\title{
Advanced Analytical Capabilities on FIB Instruments Using SIMS
}

Tom Wirtz, Olivier De Castro, Antje Biesemeier, Hung Quang Hoang and Jean-Nicolas Audinot

Luxembourg Institute of Science and Technology (LIST), Belvaux, Grevenmacher, Luxembourg

Secondary Ion Mass Spectrometry (SIMS) is an extremely powerful technique for analyzing surfaces, owing in particular to its ability to detect all elements from $\mathrm{H}$ to $\mathrm{U}$ and to differentiate between isotopes, its excellent sensitivity and its high dynamic range. SIMS analyses can be performed in different analysis modes: acquisition of mass spectra, depth profiling, 2D and 3D imaging. Adding SIMS capability to FIB instruments offers a number of interesting possibilities, including highly sensitive analytics, in-situ process control during patterning and milling, highest resolution SIMS imaging $(\sim 10 \mathrm{~nm})$, and direct correlation of SIMS data with data obtained with other analytical or imaging techniques on the same instrument, such as high resolution SE images or EDS spectra [1,2]. Past attempts of performing SIMS on FIB instruments were rather unsuccessful due to unattractive detection limits, which were due to (i) low ionization yields of sputtered particles, (ii) extraction optics with limited collection efficiency of secondary ions and (iii) mass spectrometers having low duty cycles and/or low transmission. In order to enable high-performance SIMS analysis on FIB instruments (i.e. high sensitivity, high spatial resolution, fast acquisition times, high mass resolution, etc.), we have developed a compact magnetic sector SIMS system optimized for FIB instruments. This SIMS add-on system is based on (i) specifically designed secondary ion extraction optics coupled with post-acceleration transfer optics, providing maximized extraction efficiency and transmission while keeping a finely focussed primary ion beam for highest lateral resolution, (ii) a compact floating double focusing magnetic sector mass analyser allowing operation in the DC mode at full transmission (and hence avoiding duty cycles like in TOF systems that either lead to very long acquisition times or, for a same acquisition time, intrinsically limit the sensitivity) and (iii) a specific detection system based on a continous focal plane detector allowing the detection of all masses in parallel. We installed such SIMS systems on different FIB based instruments, including the Helium Ion Microscope [3-5], a FIB-SEM DualBeam instrument and the npSCOPE instrument, which is an integrated Gas Field Ion Source enabled instrument combining SE, SIMS and STIM imaging with capabilities to analyze the sample under cryo-conditions [6]. We have demonstrated that these FIB-SIMS instruments are capable of producing (i) a full mass spectrum with mass resolution $M / \Delta M=400-500$ for each single pixel, (ii) very local depth profiles making it possible to follow the chemical composition in real time during nano-patterning in FIB for applications such as end-pointing and (iii) elemental SIMS maps with a lateral resolution down to $12 \mathrm{~nm}$ [3-5]. Furthermore, FIB-SIMS opens the way for in-situ correlative imaging combining high resolution SE images with elemental and isotopic ratio maps from SIMS [3-5]. This approach allows SIMS maps of exactly the same zone imaged in the SE mode to be acquired easily and rapidly, followed by a fusion between the SE and SIMS data sets [2]. Here, we will review the performance of the different instruments with a focus on new developments such as cryo-capabilities and new detectors allowing parallel detection of all masses, showcase methodologies for high-resolution 3D chemical imaging, present a number of examples from various fields of applications (nanoparticles, battery materials, photovoltaics, micro-electronics, tissue and sub-cellular imaging in biology, geology,...) and give an outlook on new trends and prospects [6]. 


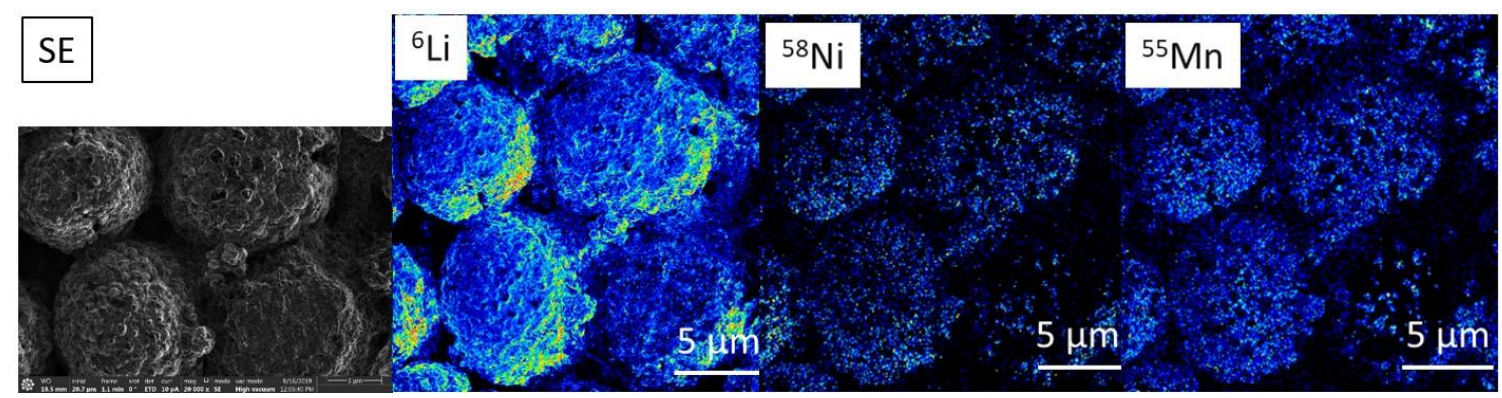

Figure 1.

\section{References}

[1] T. Wirtz, P. Philipp, J.-N. Audinot, D. Dowsett, S. Eswara, Nanotechnology 26 (2015) 434001

[2] F. Vollnhals, J.-N. Audinot, T. Wirtz, M. Mercier-Bonin, I. Fourquaux, B. Schroeppel, U. Kraushaar, V. Lev-Ram, M. H. Ellisman, S. Eswara, Anal. Chem. 89 (2017) 10702

[3] D. Dowsett, T. Wirtz, Anal. Chem. 89 (2017) 8957

[4] T. Wirtz, D. Dowsett, P. Philipp, Helium Ion Microscopy, ed. by G. Hlawacek, A. Gölzhäuser, Springer, 2017

[5] T. Wirtz, O. De Castro, J.-N. Audinot, P. Philipp, Ann. Rev. Anal. Chem. 12 (2019)

[6] This project has received funding from the European Union's Horizon 2020 Research and Innovation Programme under grant agreement No. 720964. 